\title{
Review
}

\section{Pulmonary alveolar proteinosis: from classification to therapy}

Pulmonary alveolar proteinosis (PAP) is a rare respiratory syndrome characterised by the accumulation of surfactant lipoproteins within the alveoli. According to various pathogenetic mechanisms and aetiologies, PAP is classified as primary, secondary or congenital. Primary PAP is led by a granulocytemacrophage colony-stimulating factor (GM-CSF) signalling disruption; the autoimmune form is driven by the presence of anti GM-CSF autoantibodies and represents $90 \%$ of all the PAP cases; and the hereditary form is the result of mutations in genes encoding GM-CSF receptor. Secondary PAP is associated with various diseases causing a reduction in function and/or number of alveolar macrophages. Congenital PAP emerges as a consequence of corrupted surfactant production, due to mutations in surfactant proteins or lipid transporter, or mutations affecting lung development. The clinical manifestations are various, ranging from insidious onset to acute or progressive respiratory failure, including premature death within the first days of life in neonates with congenital surfactant production disorders. The diagnostic workup includes clinical and radiological assessment (respiratory function test, high-resolution chest computed tomography), laboratory tests (anti-GMCSF autoantibodies dosage, GM-CSF serum level and GM-CSF signalling test), and genetic tests. Whole-lung lavage is the current gold standard of care of PAP; however, the therapeutic approach depends on the pathogenic form and disease severity, including GM-CSF augmentation strategies in autoimmune PAP and other promising new treatments.

\section{Educational aims}

- To update knowledge about a rare respiratory syndrome, pulmonary alveolar proteinosis, in order to promote early diagnosis and correct management.

- To highlight recent treatment options based on pathogenesis and disease severity. with various and heterogeneous aetiologies, caused by the impairment of pulmonary surfactant clearance or by abnormal surfactant production https://bit.ly/3aFpQm9
Cite as: Salvaterra E, Campo I. Pulmonary alveolar proteinosis: from classification to therapy. Breathe 2020; 16 : 200018. 


\section{Pulmonary alveolar proteinosis: a respiratory syndrome rather than a single disease}

Pulmonary alveolar proteinosis (PAP) is a rare respiratory syndrome characterised by the accumulation of surfactant lipoproteins within the alveoli leading to a variable impairment of pulmonary gas transfer and causing a broad spectrum of clinical manifestation, from exercise intolerance to hypoxaemic respiratory failure and death [1]. PAP was firstly described in 1958 and belongs is an alveolar filling disorder [2]. Basically, PAP is caused by an impairment of surfactant clearance or abnormal surfactant production, according to various pathogenetic mechanisms and different aetiologies.

At present, PAP is classified in accordance with the underlying pathogenetic mechanism as primary, secondary or congenital (table 1) [3].

\section{Primary PAP}

In primary PAP, an altered macrophage and neutrophil activation, induced by a dysfunction of granulocyte-macrophage colony-stimulating factor (GM-CSF) signalling, leads to impaired surfactant clearance and to consequent accumulation of surfactant. Primary PAP can be autoimmune or hereditary: the autoimmune form represents the $90 \%$ of all PAP cases, and the discovery of GM-CSF autoantibodies changed our clinical and experimental approach to this rare respiratory disease [4]; the hereditary form is the result of mutations involving the GM-CSF receptor (in the CSF2RA or CSF2RB genes that code for the $\alpha$ - and $\beta$-chains, respectively).

Table 1 Classification of PAP

\begin{tabular}{|c|c|}
\hline $\begin{array}{l}\text { Primary PAP: } \\
\text { GM-CSF signalling } \\
\text { disruption }\end{array}$ & $\begin{array}{l}\text { Autoimmune PAP (GM-CSF autoantibodies) } \\
\text { Hereditary PAP (mutations in genes encoding } \\
\text { GM-CSF receptor) }\end{array}$ \\
\hline $\begin{array}{l}\text { Secondary PAP: } \\
\text { reduction in } \\
\text { function and/ } \\
\text { or number } \\
\text { of alveolar } \\
\text { macrophages }\end{array}$ & $\begin{array}{l}\text { Haematological disorders } \\
\text { Malignancies } \\
\text { Immune deficiency syndromes } \\
\text { Chronic inflammatory syndromes } \\
\text { Chronic infections } \\
\text { Toxic inhalation syndromes } \\
\text { Other }\end{array}$ \\
\hline $\begin{array}{l}\text { Congenital } \\
\text { PAP: impaired } \\
\text { surfactant } \\
\text { production }\end{array}$ & $\begin{array}{l}\text { Mutations in surfactant proteins (SFTPA, SFTPB, SFTPC) } \\
\text { Mutations in lipid transporter (ABCA3) } \\
\text { Mutations affecting lung development (TTF1) }\end{array}$ \\
\hline
\end{tabular}

GM-CSF: granulocyte-macrophage colony-stimulating factor.

\section{Secondary PAP}

Secondary PAP results from various underlying conditions that can affect the number and/or the function of the alveolar macrophages. It is predominantly a consequence of haematological disorders but has also been reported in association with pharmacological immunosuppression, malignancies, chronic inflammatory conditions or environmental exposure to toxic substances [5].

\section{Congenital PAP}

Congenital PAP is determined by mutations in genes encoding surfactant proteins or proteins involved in surfactant production; as a result, the surfactant is dysfunctional and cannot fulfil its physiological roles. Rarely, the aetiology of PAP is indefinable [6].

\section{PAP: rare and rarest forms}

Despite the advances in understanding its pathophysiology, the prevalence of PAP remains poorly described. The overall prevalence has been measured to be nearly seven cases per million individuals in the general population of Japan and the USA [7, 8], where the largest population studies have been conducted. In the retrospective US epidemiological study published in 2018, McCARTHY et al. [8] described an annual prevalence of PAP of $6.87 \pm 0.33$ per million, slightly higher than the prevalence estimated in 2008 among adults in Japan (6.2 per million). Notwithstanding, PAP prevalence could be underestimated, firstly because of its insidious onset with mild, nonspecific respiratory symptoms for long periods before coming to medical attention, and second, the rarity of the disease and the corresponding lack of knowledge leads to frequent misdiagnosis. Regardless of sex, PAP prevalence increases with age in a bimodal distribution with a first peak between 45 and 54 years and a second one in patients $>75$ years [8]. Overall, primary PAP represents $90 \%$ of all PAP cases and a predominant prevalence of autoimmune PAP is uniformly reported in all major epidemiological studies [7, 8]. Furthermore, in the Japanese national registry, the median age at diagnosis was 51 years and two-thirds of the patients were men (patients $<10$ years of age were rare) [7]. In addition, according to the concurrent US study of an independent cohort of 700 PAP patients, $3 \%$ had hereditary PAP related to mutations of GM-CSF receptor [8]. Secondary PAP represents $10 \%$ of all PAP forms and is most frequently associated with haematological disorders (in particular, myelodysplastic syndromes) and the median age at diagnosis is higher than in other aetiologies[5]. Congenital PAP is the rarest and can occur in neonates, infants and children, but also in adolescents and adults $[7,8]$. 


\section{The importance of} pulmonary surfactant in all PAP forms: composition, function and homeostasis

In 1994, the discovery that knock-out mice for GM-CSF developed PAP as well as the identification in 1999 of GM-CSF autoantibodies in patients affected by autoimmune PAP (previously named idiopathic PAP) triggered intense research worldwide and completely changed the clinicaltherapeutic approach to this syndrome $[9,10]$.

More precisely, PAP is caused by an impairment of surfactant homeostasis or abnormal surfactant production. Surfactant is an amphipathic lipidprotein complex, and is normally synthesised and secreted into the alveolar space by type II alveolar epithelial cells to form a membrane-like layer [3]. Surfactant plays a pivotal role in the maintenance of lung volumes during the respiratory cycle by reducing surface tension at the air-liquid interface, thereby preventing end-expiratory alveolar collapse and atelectasis; moreover, it stabilises alveolar size, reduces elastic recoil of the lung and contributes to host defence against microbiological pathogens [11]. Alveolar surfactant is $80 \%$ composed of phospholipids (mostly phosphatidylcholine); and $10 \%$ neutral lipids, predominantly free cholesterol with traces of triglycerides and free fatty acids. The protein portion accounts for the remaining 10\%: four principals surfactant proteins have been identified (surfactant protein (SP)-A, SP-B, SP-C and $S P-D$ ), which are expressed at relatively high levels in type II cells and a small fraction of serum proteins. The production, processing, composition and removal of surfactant is finely controlled, and surfactant is finally catabolised, removed and recycled, mainly by the alveolar macrophages [12]. GM-CSF is a cytokine produced by type II alveolar epithelial cells that functions as a haematopoietic growth factor. Through binding to its specific receptors on macrophages, GM-CSF induces the phosphorylation of signal transducer and activator of transcription (STAT)5, and subsequently, the activation of multiple signalling pathways leading to the terminal differentiation of alveolar macrophages in the human lung (figure 1) [13].

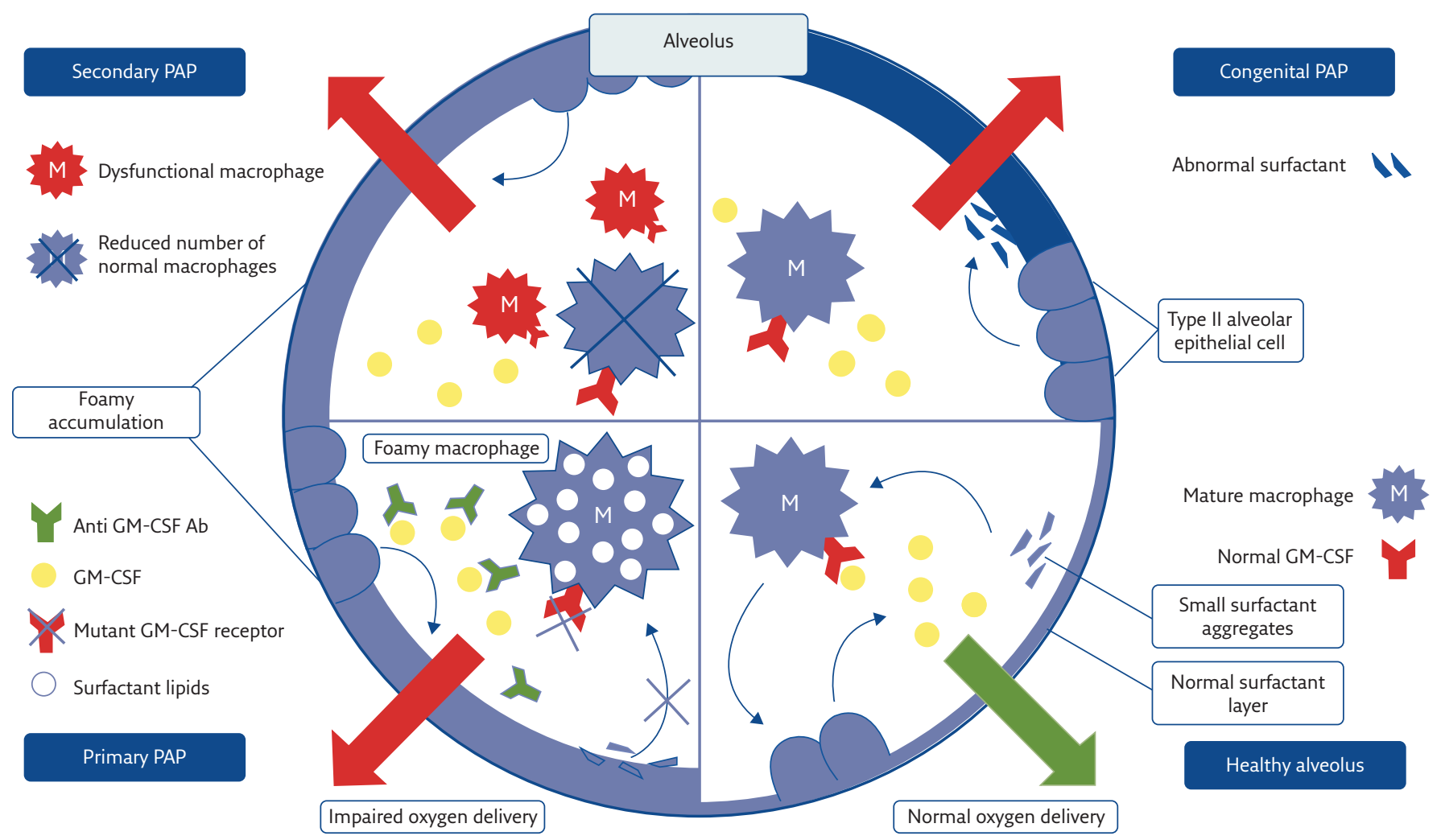

Figure 1 PAP pathogenesis. In normal surfactant homeostasis (bottom right), surfactant is synthesised by type II alveolar epithelial cells and secreted into the alveolar space to generate a thin layer. GM-CSF is a cytokine produced by type II alveolar epithelial cells that binds specific receptors on macrophages; GM-CSF induces the terminal differentiation of alveolar macrophages, allowing an effective surfactant catabolism. In primary PAP (bottom left), altered macrophage activation, due to a dysfunction in GM-CSF signalling, leads to impaired surfactant clearance and to the consequent accumulation of surfactant. In autoimmune PAP, GM-CSF signalling is inhibited by the presence of neutralising GM-CSF autoantibodies; the hereditary form is the result of mutations involving the GM-CSF receptor. In secondary PAP (top left), various underlying conditions can affect the number and/or the function of the alveolar macrophages, causing reduced surfactant clearance and surfactant accumulation within the alveoli. In congenital PAP (top right), mutations in SFTPB, SFTPC, ABCA3 and TTF1 interfere with the production of surfactant which, in turn, is ineffective and prone to accumulation. 


\section{Primary PAP}

Autoimmune PAP

Autoimmune PAP is mediated by autoantibodies targeting GM-CSF. This specific pathogenetic mechanism is supported by several lines of evidence. When PAP patient-derived neutralising autoantibodies against GM-CSF were injected into nonhuman primates, the latter developed the cardinal features of PAP [14]. Autoantibodies against GM-CSF are detectable in autoimmune PAP patients at a level $\geq 5 \mu \mathrm{g} \cdot \mathrm{mL}^{-1}$, while in hereditary, secondary or congenital PAP patients, in patients with other lung disease and in healthy subjects, the GM-CSF autoantibody level is under this threshold [15]. In fact, low levels of GM-CSF autoantibodies are ubiquitously present in people without autoimmune PAP [16] Hence, the "critical threshold" value of GM-CSF autoantibody concentration $\geq 5 \mu \mathrm{g} \cdot \mathrm{mL}^{-1}$ is able to promote GM-CSF sequestration and degradation, disrupting GM-CSF-stimulated functions in alveolar macrophages and blood leukocytes [16]. However, GM-CSF autoantibody levels, as currently measured, do not correlate with disease severity [17].

The reduction in surfactant-degrading capability of alveolar macrophages, as a consequence of GM-CSF biological activity inhibition, leads to the accumulation of surfactant and ensuing respiratory insufficiency. Furthermore, GM-CSF neutralisation results in impaired antimicrobial activity of neutrophils with increased risk of infections $[17,18]$.

The aetiology of the autoimmune response has been evaluated by characterising GM-CSF autoantibodies. These are composed of polyclonal immunoglobulin $\mathrm{G}$ targeting multiple epitopes of GM-CSF molecules with high binding affinity, and the capability to neutralise GM-CSF at high concentrations and to effectively block GM-CSF signalling in vivo. The demonstration of GM-CSF autoantibody polyclonality precludes that a single clone of autoantibodies is responsible for the pathogenesis in each patient. Immune targeting of multiple different GM-CSF epitopes indicates that autoantibody formation is steered by GM-CSF and not by a pathogen-related, cross-reacting epitope [19].

\section{Hereditary PAP}

Hereditary PAP is caused by the disruption of GM-CSF signalling because of homozygous or compound heterozygous mutations in the CSF2RA and CSF2RB genes, which lead to reduced protein expression on the cell surface $[20,21]$. The heterogeneity of disease severity across family members with identical mutations suggests that other factors may be involved in addition to GM-CSF signalling. Hereditary PAP is clinically, physiologically and histologically indistinguishable from autoimmune PAP [22].

\section{Secondary PAP}

A wide heterogeneity of diseases can be associated twith secondary PAP [1]. The mechanisms predisposing secondary PAP can be identified as reduced numbers or function of alveolar macrophages, resulting in an impaired capacity to clear surfactant from the lung [5]. Systemic disorders accompanying secondary PAP include malignant and nonmalignant haematological diseases, nonhaematological malignancies, immune deficiency syndromes, chronic inflammatory syndromes, and chronic infections. Among the haematological disorders, chronic myeloid leukaemia and myelodysplastic syndromes are predominately associated with PAP [3]. Furthermore, immune deficiency syndromes can be associated with secondary PAP, like thymic alymphoplasia (deficiency of lymphocytes in the thymus), immunoglobulin $A$ deficiency, immunosuppression as result of solid organ transplantation and AIDS [3].

\section{Congenital PAP}

Congenital PAP occurs as a respiratory disease that can emerge in neonates and children but also in adults, is characterised by different levels of surfactant accumulation, and is always associated with pulmonary fibrosis [23]. Mutations in SFTPB (SP-B), SFTPC (SP-C), ABCA3 and TTF1 have been associated with congenital PAP, as they lead to the disruption of the production and the function of surfactant. SP-B and SP-C are hydrophobic peptides located at the alveolar air-liquid interface. Homozygous mutations for recessive loss-offunction mutations in SFTPB were found in infants who developed respiratory failure and died shortly after birth. Individuals heterozygous for recessive loss-of-function SFTPB alleles, instead, have normal lung function. Autosomal dominant mutations in SFTPC are linked with interstitial lung disease at all ages.

$A B C A 3$ is a member of the ABCA transporter family known to be involved in transmembrane transport of endogenous lipids; it is expressed in the type II alveolar cells and is mostly detected at the limiting membrane of the lamellar bodies, where the surfactant complex is assembled, processed and stored [6, 23]. Fatal surfactant deficiency and premature death also occur in infants homozygous for recessive loss-of-function mutations in $A B C A 3$. While some other $A B C A 3$ mutations result in dysfunctional $A B C A 3$ and, consequently, in dysfunctional surfactant deficient in phosphatidylcholine, causing chronic respiratory disease in older children and adults

The transcription factor TTF1 is essential for lung development. Haploinsufficiency of TTF1 causes a complex phenotype in neonates that can include hypothyroidism, brain abnormalities and acute and chronic lung disease [24]. 


\section{When to suspect PAP: clinical, radiological and functional aspects}

\section{Signs and symptoms}

Autoimmune PAP usually occurs in adults in the third to fifth decade of life with an insidious onset (one third of patients with autoimmune PAP are asymptomatic), and is characterised mainly by exertional dyspnoea, eventually accompanied by nonspecific respiratory symptoms, like cough and/ or production of white frothy sputum, or systemic symptoms such as fatigue and/or weight loss (table 2). This unclear presentation heads to long periods before evaluation or initial diagnosis [3, 7]. Hereditary PAP presents similarly to autoimmune PAP, except that the age of onset is typically, but not always, in late infancy or childhood [22]. Secondary PAP arises as cough, dyspnoea and gas exchange impairment during other ongoing disease or after environmental exposure [5]. Patients with congenital PAP present variably, depending on the specific gene mutation, with onsets ranging from premature death within the first days of life to adult debut of symptoms [6, 23, 24]. Aside from neonatal and paediatric onset in congenital PAP, physical examination is generally unremarkable; however, crackles and cyanosis have been reported in a small proportion of patients. Digital clubbing is rarely a manifestation of PAP, while fever and haemoptysis are uncommon and generally associated with superimposed infections. Nevertheless, fever can be a manifestation of PAP itself. Considering these confounding signs and symptoms, patients are usually misdiagnosed with pneumonia, on the basis of radiological findings, or asthma (especially in children), on the basis of nonspecific symptoms until the failure to respond to "appropriate" therapy. In such cases, an accurate diagnosis is delayed, on average, by 18 months [3].

\section{Radiological findings}

Chest radiography commonly demonstrates diffuse bilateral symmetrical infiltrates in a perihilar distribution, which can progress to confluent infiltrates involving all the five lobes [25].

Table 2 PAP clinical manifestations

\begin{tabular}{lr}
\hline No symptoms & $31.4 \%$ \\
Exertional dyspnoea & $39.0 \%$ \\
Cough & $9.9 \%$ \\
Dyspnoea and cough & $10.9 \%$ \\
Dyspnoea and sputum & $1.3 \%$ \\
Dyspnoea, cough and sputum & $2.2 \%$ \\
Other & $4.0 \%$ \\
\hline
\end{tabular}

High-resolution computed tomography (HRCT) of the chest is mandatory if a suspicion of PAP exists, to identify the peculiar radiological pattern that is characterised by interlobular septal thickening associated with patchy ground glass, known as "crazy paving", referring to the polygonal appearance of the secondary pulmonary lobules within interspersed ground-glass attenuation (figure $2 \mathrm{a}$ ). Areas of consolidation with air bronchograms can be present in addition to the ground-glass opacification [26]. However, the crazy paving pattern is not specific for PAP and can be found in a variety of other lung diseases such as infections, malignancies, pulmonary oedema, alveolar haemorrhage, organising pneumonia, acute respiratory distress syndrome, Pneumocystis pneumonia and lipoid pneumonia [26]. Fibrosis on HRCT at baseline or at follow-up predicts a poor prognosis. Pleural effusion, enlargement of mediastinal lymph nodes and evidence of air trapping are not characteristic, and should lead to alternate or concomitant diagnosis. Pulmonary nodules are also atypical and should be assessed for malignancy or infection [27].

\section{Lung function testing}

Lung volumes are generally within normal limits in the early stages of the disease, a reduction of forced vital capacity and total lung capacity, consistent with restrictive ventilatory pattern, can be found in patients with severe disease [7]. However, the diffusing capacity of the lung for carbon monoxide $\left(D_{\mathrm{LCO}}\right)$ is frequently reduced and this impairment correlates with disease severity [1]. Furthermore, the alveolar-arterial oxygen gradient is often increased, indicating alveolar defective gas exchange and being a better criteria than $D_{\mathrm{Lco}}$ to determine disease activity $[1,2]$. Oxygen desaturation on exercise and severe hypoxaemia at rest reflect major disease involvement, and are useful indicators for the need of treatment [1, 2].

\section{From clinical suspicion to PAP diagnosis, step by step}

A clinical picture of slowly progressive nonspecific respiratory symptoms in association with typical chest HRCT findings should suggest a diagnosis of PAP (figure 3).

Most routine laboratory tests are usually within normal range, except lactate dehydrogenases levels, which can frequently be high [1]. Several circulating biomarkers have been identified to correlate variably with disease severity, such as serum tumour antigens (carcinoembryonic antigen, carbohydrate antigen 19.9, CYFRA 21-1 and neuron-specific enolase), lung epithelium-derived proteins (mucin, KL-6, SP-A, SP-B and SP-D), chemokines and cytokines (CCL-2), and chitinase 3-like protein 1 (also known as YKL40) [28]. However, none of these 


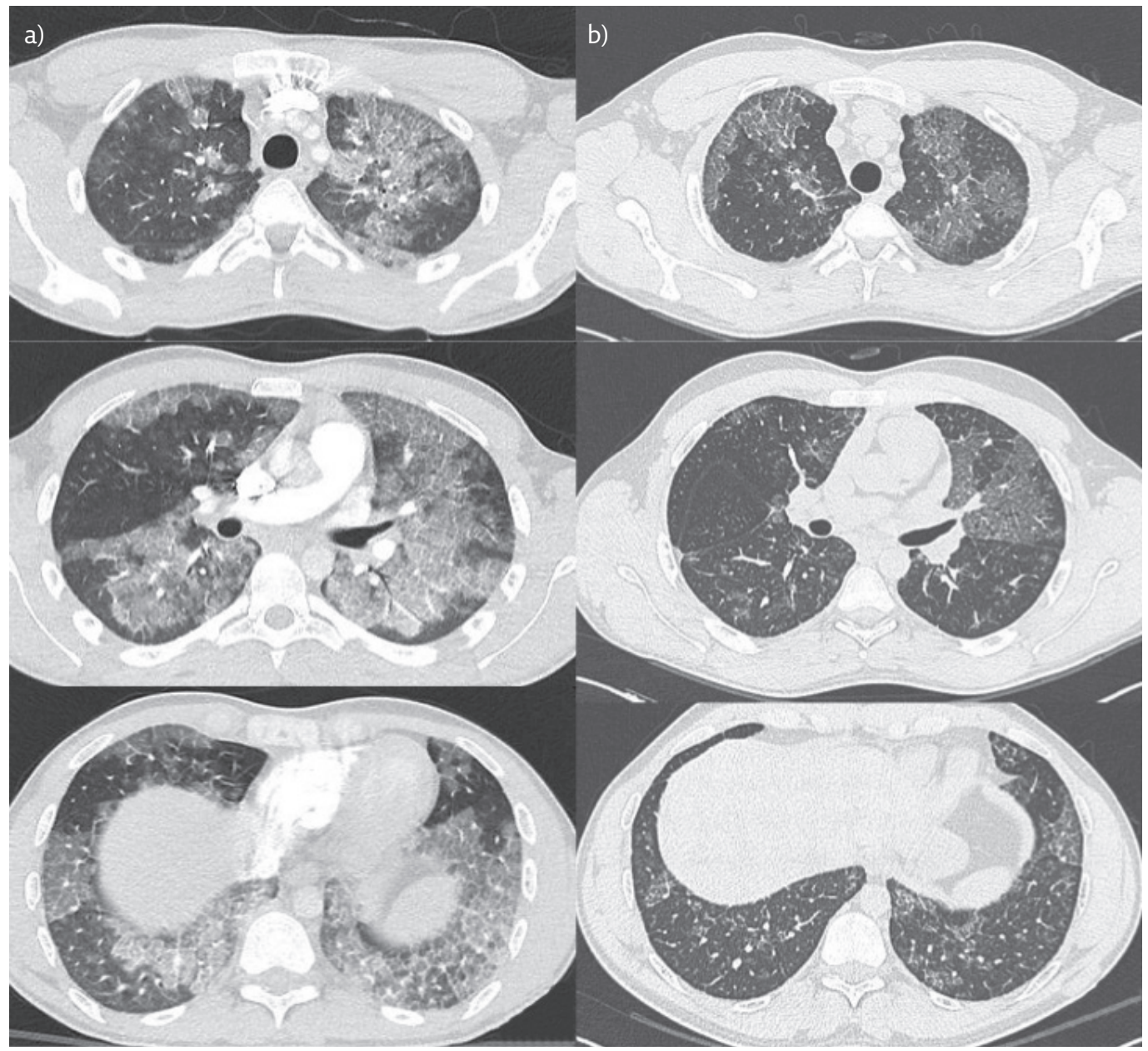

Figure 2 Chest HRCT findings in PAP. a) Interlobular septal thickening within interspersed ground glass ("crazy paving" pattern) is spread symmetrically to all five lobes with a perihilar predominance. b) After whole-lung lavage treatment, the crazy paving pattern is still appreciable but with reduced distribution and lower alveolar infiltration.

biomarkers is specific or diagnostic for PAP and they still are a matter of research.

\section{Endoscopic confirmation}

Flexible bronchoscopy with BAL is the first step to obtaining a definite diagnosis of PAP syndrome; BAL fluid appears opaque and milky, and usually shows large amounts of sediment. The microscopic examination demonstrates acellular globules, which are basophilic after May-Grünwald-Giemsa and periodic acid-Schiff (PAS) staining, as well as foamy alveolar macrophages positive to oil-red-O staining and a substantial amount of cell debris that stains only weakly with PAS staining [1, 29]. In addition, bronchoscopy is useful to isolate certain microorganisms that can mimic PAP or to identify intercurrent infection. Neither transbronchial nor surgical lung biopsy is indicated on account of the substantial false-negative rate [30].

\section{Classification}

After identifying PAP syndrome, it is necessary to proceed with other diagnostic tools in order to determine the specific PAP form in observation. An accurate medical, occupational and environmental exposure history has to be collected in order to identify any cause of secondary PAP.

\section{GM-CSF autoantibody test}

Considering that autoimmune PAP is the most frequent cause of PAP syndrome, serum GM-CSF autoantibody titration should be the first diagnostic test $[7,15,30]$. The identification of high levels of GM-CSF autoantibodies in serum is diagnostic for autoimmune PAP; autoantibody levels to define autoimmune PAP should be higher than the critical threshold of $5 \mu \mathrm{g} \cdot \mathrm{mL}^{-1}[4,15]$. Low levels of GM-CSF autoantibodies can be detectable (usually $<1 \mu \mathrm{g} \cdot \mathrm{mL}^{-1}$ ) in serum from healthy people and patients with malignancies, inflammatory conditions and secondary PAP [16]. This diagnostic test shows a sensitivity and specificity of $100 \%$ for autoimmune PAP [15], but is practicable in few laboratories worldwide, in the USA, Japan, Germany and China, with affiliated clinical centres in other countries (e.g. Italy and the Netherlands). Current information about the availability of diagnostic 


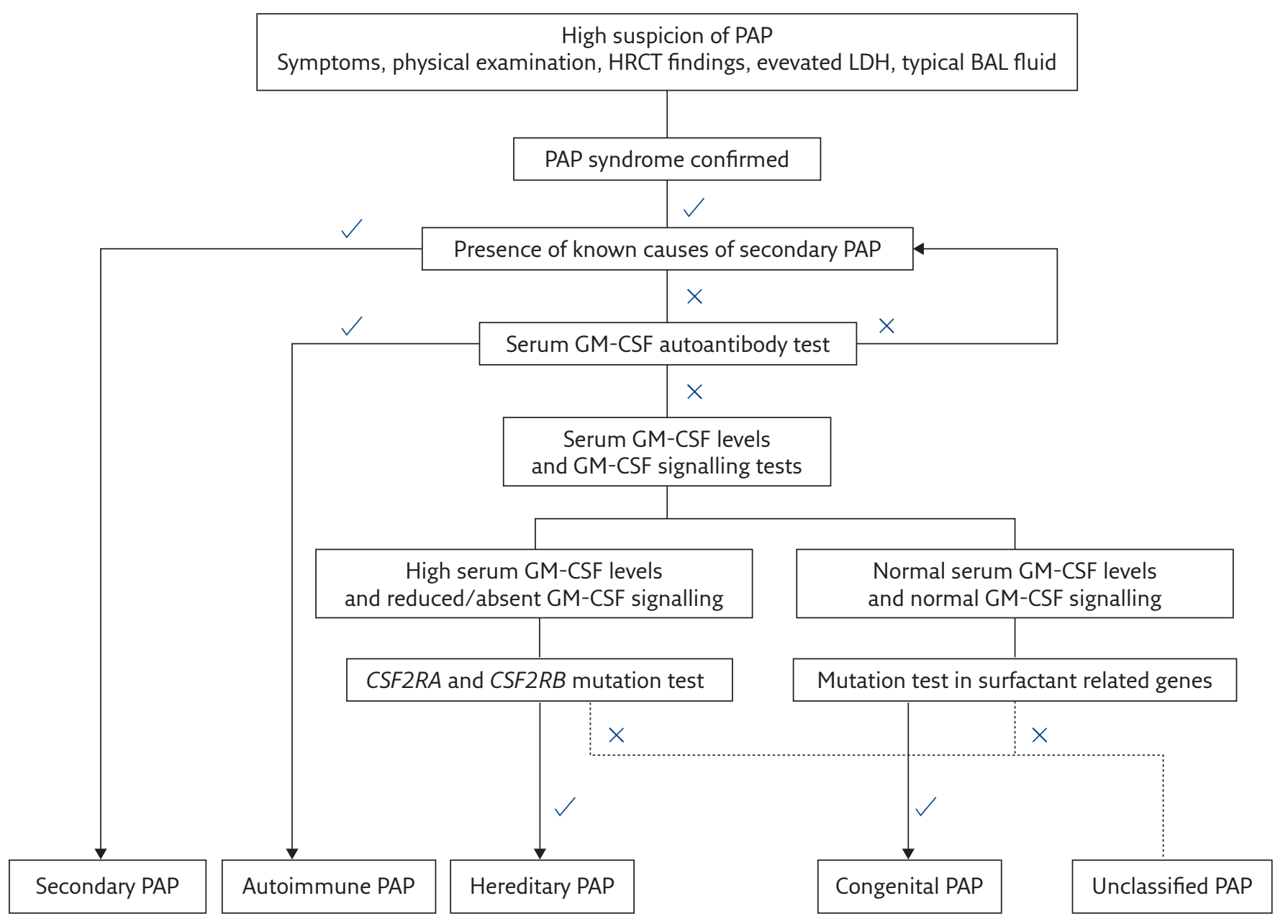

Figure 3 Diagnostic algorithm of PAP syndrome. PAP can be suspected based on a congruent clinical picture, compatible chest HRCT findings and an evocative $B A L$ fluid. BAL fluid cytological analysis can confirm the diagnosis of PAP syndrome; subsequently, the diagnostic effort is directed to the identification of the specific PAP-causing disease. In the absence of a clear PAP-causing disease, GM-CSF autoantibody measurement should be performed to identify autoimmune PAP, the most frequent aetiology of PAP. Low levels of GM-CSF autoantibodies exclude the diagnosis of autoimmune PAP and dictate further investigations: accurate research of possible secondary PAP-causing disease, serum GM-CSF measurement and GM-CSF signalling test. High levels of serum GM-CSF indicate GM-CSF receptor dysfunction, highlighting hereditary PAP, which can be confirmed by the GM-CSF signalling test and specific gene analysis. Normal levels of serum GM-CSF and a GM-CSF signalling test within normality suggest the presence of congenital PAP that should be assessed with specific genetic tests [4]. LDH: lactate dehydrogenase.

testing for PAP is available from the PAP Foundation and EuPAPNet, the European network for PAP.

In the absence of GM-CSF autoantibodies, or the presence of low levels of GM-CSF autoantibodies in patients with a confirmed PAP syndrome without any clear cause of secondary PAP, should prompt an GM-CSF concentration test and GM-CSF signalling test in an effort to orientate specific gene analysis.

Serum GM-CSF concentration and blood-based GM-CSF signalling tests

Serum GM-CSF concentration testing should be considered for patients with PAP syndrome with normal levels of GM-CSF autoantibodies and if secondary PAP has been discounted. Serum GM-CSF concentration is elevated $\left(>10 \mathrm{pg} \cdot \mathrm{mL}^{-1}\right)$ in patients with hereditary PAP caused by CSF $2 R A$ or CSF2RB mutations (owing to reduced clearance by dysfunctional receptors); conversely, it is typically undetectable or below the lower limit of quantification $\left(7 \mathrm{pg} \cdot \mathrm{mL}^{-1}\right)$ in healthy individuals [31]. This test must be considered a screening test because GM-CSF can be increased during infections in patients without hereditary PAP. GM-CSF signalling can be evaluated by quantifying the level of intracellular phosphorylated STAT5 or cell-surface CD11b in neutrophils in response to GM-CSF [32]. Positive results require subsequent gene analysis, looking for CSF2RA or CSF2RB mutations.

Genetic testing for causes of congenital and secondary PAP

These tests should involve screening for mutations in genes required for surfactant production (SFTPA, SFTPB, SFTPC, ABCA3 or TTF1) [23, 33]. Mutations in SFTPB and $A B C A 3$ drive a significant disruption of surfactant production and packing, usually resulting in respiratory failure in term newborn infants. Mutations in SFTPC and SFTPA usually emerge clinically later in infancy or in adulthood 
[6, 23]. Mutations in $A B C A 3$ can cause a wide clinical spectrum with varying severity, ranging from neonatal respiratory failure and death during the first days or months of life to interstitial lung disease at all ages [6]. A variety of findings are observed on histopathological examination of lung tissue, including the accumulation of lipoproteinaceous material in the airspaces and varying amounts of interstitial thickening [34]. Finally, it is also important to screen for mutation in genes associated with the development of secondary PAP [3].

\section{PAP clinical course}

According to several large cross-sectional cohort studies, three clinical evolution patterns have been associated with autoimmune PAP: progressive deterioration, stable but unremitting disease and spontaneous resolution [1, 3]. The Japanese national PAP registry study reported no deaths over the 5-year period of follow-up among autoimmune PAP patients [7]. The clinical course of hereditary PAP is comparable to autoimmune PAP (both the diseases are driven by the disruption of GM-CSF signalling) but the available data are still insufficient to estimate the overall survival [20-22]. However, the clinical picture of a patient affected by secondary PAP is peculiar and is characterised by a remarkably worse prognosis, a median survival $<20$ months and 2 -year survival that has been reported in only $40 \%$ of cases [5]. Notably, the poor survival seems to be related to the underlying disease rather than PAP [3, 5]. In congenital PAP, the clinical evolution is highly affected by the involved gene and gene variant. Since the inhibition of GM-CSF biological activity is responsible for macrophage immaturity, which precludes normal surfactant homeostasis and effective control of infection, in patients affected by PAP, there is a higher incidence of pulmonary or systemic infections, caused by common or opportunistic pathogens [1]. Infections account for $18-20 \%$ of deaths related to PAP. They are quite common complication, $13 \%$ of cases, and can occur at disease onset or anytime during the clinical course of the disease. Opportunistic pathogens can include Nocardia, Mycobacterium or fungi, mainly Aspergillus species [1]. Pulmonary fibrosis is reported to be associated with any PAP form, rarely and only in advanced disease in patients with primary PAP; congenital PAP is usually accompanied by marked parenchymal distortion, fibrosis and respiratory insufficiency [35, 36]. Hence, investigations on the potential fibrotic evolution must be part of the routine follow-up in PAP patients.

\section{Different PAP forms, different therapies}

The therapeutic approach to PAP is strictly related to the pathogenic form and disease severity. Treatment options range from the watchful waiting for patients with asymptomatic autoimmune PAP to whole-lung lavage (WLL) therapy, the current gold standard of care.

\section{Whole-lung lavage}

WLL is an invasive procedure that can only be performed properly in specialised centres. It is the current standard of treatment for patients affected by primary PAP and in some causes of secondary PAP (but not in congenital PAP) [3]. However, WLL is not a standardised procedure and international consensus documents are lacking. In 2016, a clinical practice survey, based on a questionnaire, was globally conducted and included 27 centres performing WLL in paediatric and/or adult PAP patients [37]. WLL is an invasive procedure almost universally performed under general anaesthesia, in an intensive care unit, by using a double-lumen endobronchial intubation. During the procedure, one lung undergoes the lavage, while the other is selectively ventilated (figure 4). The interval between the first lavage and the second session varies among centres, with an interval of 1-2 weeks in $\sim 50 \%$ of centres. Homogenous across centres is the use of saline warmed to $37^{\circ} \mathrm{C}$, the drainage of lung lavage fluid by gravity and the indications for WLL therapy. Specific indications include a decline in arterial oxygen tension $\left(\mathrm{PaO}_{2}\right)$ at rest, worsening of lung disease severity on radiological evaluation, and decline in diffusing capacity of the lung for carbon monoxide $\left(D_{\mathrm{LCO}}\right)$, forced vital capacity or resting oxygen saturation by pulse oximetry, or an increase in respiratory symptoms. The choice of the first lung to be lavaged differs between centres: $50 \%$ of centres base the choice on imaging, whereas 50\% always start with the left lung due to its smaller size. Patient position during the procedure is widely discordant as well, as in $50 \%$ of centres the patient lies in the supine position whereas other possible positions are the full lateral position at $90^{\circ}$ or a moderate lateral position at $30-45^{\circ}$ inclination. The amount of fluid used to perform the WLL is debated: a general consensus exists on the single aliquot of fluid for lavage ( $800 \mathrm{~mL}$ warm saline in adults) but the total amount of saline infused per lung ranges from 5 to $40 \mathrm{~L}$, with an average of $15.4 \mathrm{~L}$ per lung. In order to improve the therapeutic effect, most (14 out of 20) centres use chest percussion to emulsify the PAP sediment but a general consensus does not exist either for the method (manual or mechanical) or for the timing. Other issues still under discussion include contraindications, methods and timing of follow-up, timing of extubation, lung isolation, and lavage methods for small children. Despite the different procedural options, the international survey on WLL found this practice to be safe and effective as therapy for PAP (figure $2 b$ ), with a low rate of procedure-related morbidity $(-18 \%$, including, in order of decreasing frequency, fever, spillage of saline into the ventilated lung, worsened hypoxaemia, superinfections and pneumothorax) [37]. 


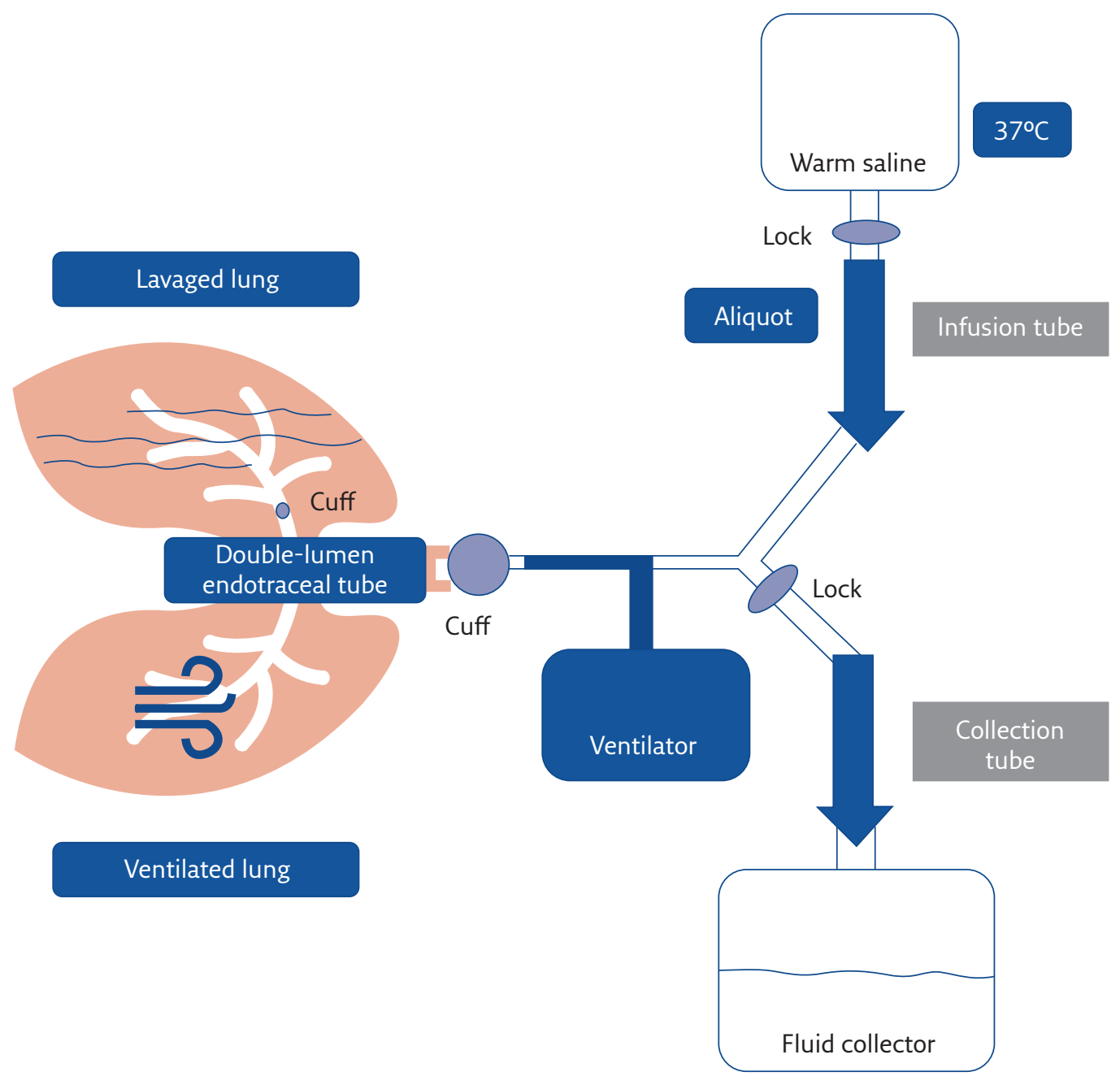

Figure 4 WLL schematic procedure. WLL is an invasive procedure almost universally performed under general anaesthesia, in an intensive care unit. In this example, the patient lies in the full lateral position; selective ventilation is assured by using a double-lumen endobronchial intubation. The lung above undergoes the lavage, while the other is selectively ventilated; an aliquot of warm saline is infused by gravity into the nonventilated lung and the collection tube is clamped. Subsequently, the aliquot is drained from the lavaged lung and received by gravity by the fluid collector. These passages are repeated until the collected fluid turns clears or until achievement of the programmed total infusion volume for each lung. Chest percussion can be associated during the procedure in order to emulsify the surfactant sediment.

\section{Autoimmune PAP therapeutic strategies}

\section{GM-CSF supplementation}

The first approach to GM-CSF augmentation as a therapy for autoimmune PAP was in the late 1990s, by subcutaneous administration of recombinant GM-CSF; consequently, improvements were reported in $\sim 50 \%$ of case studies, with a variable response among patients [3, 38]. Moreover, the administration of aerosolised recombinant GM-CSF in autoimmune PAP demonstrated better results (76.5\% versus $48.4 \%$ ) if compared with the subcutaneous route [39]. During inhaled GM-CSF therapy, the local deposition of the drug within the alveoli permits a direct activity against neutralising autoantibodies. Furthermore, inhaled GM-CSF was not associated to the bone marrow effects reported with the subcutaneous administration. A recent meta-analysis [40] of the studies of inhaled and subcutaneous GM-CSF therapy in autoimmune PAP patients showed that the pooled response rate of GM-CSF therapy ( $81 \%)$ was not inferior to WLL therapy in terms of pulmonary function tests, disease severity score and 6-min walking test. Moreover, they showed the superiority of inhaled versus subcutaneous GM-CSF therapy in term of disease-free relapse ( $89 \%$ versus $71 \%, \mathrm{p}=0.023), \mathrm{PaO}_{2}$ increase $(21.02$ versus $8.28 \mathrm{mmHg}, \mathrm{p}<0.001)$ and alveolar-arterial oxygen difference improvement (19.63 versus $9.15 \mathrm{mmHg}, \mathrm{p}<0.001)$. Although inhaled GM-CSF might be a promising therapeutic option for PAP, the data supporting GM-CSF supplementation as first-line treatment are not conclusive, but we believe that the ongoing multicentre phase II/ III trial can offer the possibility of a large-scale treatment of these patients [39]. 


\section{Self-assessment questions}

1. PAP is caused by various underlying pathogenetic mechanisms. Which is the most frequent form?
a. Hereditary PAP
b. Autoimmune PAP
c. Secondary PAP
d. Congenital PAP

2. PAP is caused by the accumulation of surfactant lipoproteins within the alveoli. Which of the following pathogenetic mechanism is at the base of autoimmune PAP?
a. The presence of anti GM-CSF receptor autoantibodies
b. The inflammation related to autoimmune disease
c. The presence of high levels of anti GM-CSF autoantibodies
d. The presence of high serum levels of GM-CSF

3. Various treatment options based on aetiology have been proposed in PAP, but which of the following therapeutic approaches is currently the gold standard of care?
a. Inhaled GM-CSF
b. WLL
c. Corticosteroids
d. Bone marrow transplantation

4. What is the most promising route to deliver GM-CSF therapy in autoimmune PAP patients?
a. Subcutaneous
b. Inhaled
c. Oral
d. Intravenous

develop either respiratory infections, graft versus host disease or drug toxicity. Alternatively, the transplantation of genetically corrected pulmonary macrophages seems to be a promising method to generate abundant functional macrophages that does not require an immunosuppressive strategy. Moreover, the use of advanced methods of genome engineering for targeted editing could guarantee an efficient and safe gene correction, which could be applied to stem cell-based gene therapy [43].

\section{Novel approach: targeting lipids homeostasis}

It is known that lipids, mainly cholesterol, are the major determinants of surfactant fluidity, which is critical for surface tension reduction. Moreover, cholesterol was identified to be the most abundant material accumulating within the alveolar macrophages in PAP patients, leading to an altered cholesterol/phospholipid ratio. In addition, a recent study has identified cholesterol as a possible target for a new therapeutic approach in PAP [44]. Two autoimmune PAP cases were reported to significantly improve after statin therapy. Statin treatment was able to reduce cholesterol content by $40 \%$ in autoimmune PAP foamy alveolar macrophages when compared with paired control cells. Furthermore, Csf2 $\mathrm{rb}^{-1-}$ mice treated in vivo with statin therapy, by oral administration for 6 weeks, demonstrated diminished BAL fluid turbidity, and decreased cholesterol concentration in BAL fluid and alveolar macrophages; more precisely, statin therapy seems to ameliorate the cholesterol efflux from Csf2 $\mathrm{rb}^{-/-}$macrophages ex vivo and the lung disease in Csf2 $\mathrm{rb}^{-/-}$mice in vivo [44].

The discovery of GM-CSF autoantibodies as the causative agents for autoimmune PAP led to the use of therapeutic approaches affecting their production and/or serum levels. Plasmapheresis to remove the autoantibodies and B-lymphocyte depletion using rituximab (an anti-B-cell monoclonal antibody) have been attempted [41, 42]; however, further studies are required to demonstrate their potential utility.

\section{Hereditary PAP therapeutic strategies}

WLL is rarely used as a treatment for hereditary PAP due to the difficulty of performing the procedure in paediatric patients [43]. Gene therapy-based approaches have been proposed but preclinical toxicology studies are mandatory before testing gene transfer in hereditary PAP patients. Transplantation of haematopoietic stem cells has been attempted in order to restore GM-CSF-dependent signalling in mice, but despite the positive results gained, this procedure required aggressive immunosuppression and myeloablation, which predisposed the patient to

\section{Conclusions}

Although our knowledge about PAP pathogenesis has greatly increased after the discovery of the role of GM-CSF, several questions still influence decisions on patient management. Early diagnosis and subsequent appropriate management could result in a marked clinical improvement for the affected patient. More precisely, in this review, we propose an algorithm that could drive clinicians towards the identification of the correct PAP form. In addition, we suggest PAP patient referral to national centres, where standard therapy and new therapeutic strategies are available. For the time being, interventional clinical trials in pulmonary alveolar proteinosis are currently ongoing, showing the feasibility of aerosolised recombinant GM-CSF for the treatment of autoimmune PAP. Finally, recent findings on lipids homeostasis within PAP alveolar macrophages are leading to new promising approaches. 


\section{Key points}

- Pulmonary alveolar proteinosis (PAP) is a rare respiratory syndrome characterised by the accumulation of surfactant lipoproteins within the alveoli, caused by an impairment of surfactant clearance or abnormal surfactant production, leading to variable impairment of the respiratory function.

- PAP is classified, according to various pathogenetic mechanisms, as primary, secondary or congenital; primary autoimmune form represents $90 \%$ of all PAP cases.

- High-resolution computed tomography of the chest shows a particular radiological pattern that is characterised by interlobular septal thickening associated with patchy ground glass, known as "crazy paving".

- Whole-lung lavage is the current gold standard of care of PAP; however, the therapeutic approach is strictly related to the pathogenic form and disease severity, including granulocyte-macrophage colonystimulating factor augmentation strategies in autoimmune PAP and other promising new treatments.

\section{Affiliations}

\section{Elena Salvaterra', Ilaria Campo²}

${ }^{1}$ Dept of Internal Medicine, University of Pavia, Pavia, Italy. ${ }^{2}$ Pneumology Unit, IRCCS Policlinico San Matteo Hospital Foundation, Pavia, Italy.

\section{Conflict of interest}

E. Salvaterra has nothing to disclose. I. Campo has nothing to disclose.

\section{References}

1. Seymour JF, Presneill JJ. Pulmonary alveolar proteinosis: progress in the first 44 years. Am J Respir Crit Care Med 2002; 166: 215-235.

2. Rosen SH, Castleman B, Liebow AA. Pulmonary alveolar proteinosis. N EnglJ Med 1958; 258: 1123-1142.

3. Trapnell BC, Nakata K, Bonella F, et al. Pulmonary alveolar proteinosis. Nat Rev Dis Primers 2019; 5: 16.

4. Uchida K, Beck DC, Yamamoto T, et al. GM-CSF autoantibodies and neutrophil dysfunction in pulmonary alveolar proteinosis. N EnglJ Med 2007; 356: 567-579.

5. Ishii $H$, Tazawa $R$, Kaneko C, et al. Clinical features of secondary pulmonary alveolar proteinosis: pre-mortem cases in Japan. Eur RespirJ 2011; 37: 465-468.

6. Nogee LM. Genetic mechanisms of surfactant deficiency. Biol Neonate 2004; 85: 314-318.

7. Inoue Y, Trapnell BC, Tazawa R, et al. Characteristics of a large cohort of patients with autoimmune pulmonary alveolar proteinosis in Japan. Am J Respir Crit Care Med 2008; 177: 752-762.

8. McCarthy C, Avetisyan R, Carey BC, et al. Prevalence and healthcare burden of pulmonary alveolar proteinosis. Orphanet J Rare Dis 2018; 13: 129

9. Stanley E, Lieschke GJ, Grail D, et al. Granulocyte/macrophage colony-stimulating factor-deficient mice show no major perturbation of hematopoiesis but develop a characteristic pulmonary pathology. Proc Natl Acad Sci UA 1994; 91: 5592-5596.

10. Tanaka N, Watanabe J, Kitamura T, et al. Lungs of patients with idiopathic pulmonary alveolar proteinosis express a factor which neutralizes granulocyte-macrophage colony stimulating factor. FEBS Lett 1999; 442: 246-250.
11. Serrano AG, Perez-Gil J. Protein-lipid interactions and surface activity in the pulmonary surfactant system. Chem Phys Lipids 2006; 141: 105-118.

12. Perez-GilJ, Weaver TE. Pulmonary surfactant pathophysiology: current models and open questions. Physiology (Bethesda) 2010; 25: 132-141.

13. Hansen $G$, Hercus TR, McClure BJ, et al. The structure of the GM-CSF receptor complex reveals a distinct mode of cytokine receptor activation. Cell 2008; 134: 496-507.

14. Sakagami T, Beck D, Uchida K, et al. Patient-derived granulocyte/ macrophage colony-stimulating factor autoantibodies reproduce pulmonary alveolar proteinosis in nonhuman primates. Am J Respir Crit Care Med 2010; 182: 49-61.

15. Uchida K, Nakata K, Carey B, et al. Standardized serum GM-CSF autoantibody testing for the routine clinical diagnosis of autoimmune pulmonary alveolar proteinosis. J Immunol Methods 2014; 402: 57-70.

16. Bendtzen K, Svenson M, Hansen MB. GM-CSF autoantibodies in pulmonary alveolar proteinosis. N Engl/ Med 2007; 356: 2001.

17. Seymour JF, Doyle IR, Nakata K, et al. Relationship of antiGM-CSF antibody concentration, surfactant protein $A$ and $B$ levels, and serum LDH to pulmonary parameters and response to GM-CSF therapy in patients with idiopathic alveolar proteinosis. Thorax 2003; 58: 252-257.

18. Piccoli L, Campo I, Fregni CS, et al. Neutralization and clearance of GM-CSF by autoantibodies in pulmonary alveolar proteinosis. Nat Commun 2015; 6: 7375.

19. Wang Y, Thomson CA, Allan LL, et al. Characterization of pathogenic human monoclonal autoantibodies against GM-CSF. Proc Natl Acad Sci USA 2013; 110: 7832-7837.

\section{Suggested answers}

1. b.

2. c.

3. b.

4. b. 
20. Suzuki T, Sakagami T, Rubin BK, et al. Familial pulmonary alveolar proteinosis caused by mutations in CSF2RA. J Exp Med 2008; 205: 2703-2710.

21. Suzuki T, Maranda B, Sakagami T, et al. Hereditary pulmonary alveolar proteinosis caused by recessive CSF2RB mutations. Eur RespirJ 2011; 37: 201-204.

22. Suzuki T, Sakagami T, Young LR, et al. Hereditary pulmonary alveolar proteinosis: pathogenesis, presentation, diagnosis, and therapy. Am J Respir Crit Care Med 2010; 182 1292-1304.

23. Whitsett JA, Wert SE, Weaver TE. Diseases of pulmonary surfactant homeostasis. Annu Rev Pathol 2015; 10: 371-393.

24. Iwatani N, Mabe H, Devriendt K, et al. Deletion of NKX2.1 gene encoding thyroid transcription factor- 1 in two siblings with hypothyroidism and respiratory failure. J Pediatr 2000 137: 272-276.

25. Lee KN, Levin DL, Webb WR, et al. Pulmonary alveolar proteinosis: high-resolution $\mathrm{CT}$, chest radiographic, and functional correlations. Chest 1997; 111: 989-995.

26. Johkoh T, Itoh H, Muller NL, et al. Crazy-paving appearance at thin-section CT: spectrum of disease and pathologic findings. Radiology 1999; 211: 155-160

27. Holbert JM, Costello P, Li W, et al. CT features of pulmonary alveolar proteinosis. AJR Am J Roentgenol 2001; 176 1287-1294.

28. McCarthy C, Kokosi M, Bonella F. Shaping the future of an ultrarare disease: unmet needs in the diagnosis and treatment of pulmonary alveolar proteinosis. Curr Opin Pulm Med 2019 25: $450-458$

29. Costabel U, Guzman J, Bonella F, et al. Bronchoalveolar lavage in other interstitial lung diseases. Semin Respir Crit Care Med 2007; 28: 514-524.

30. McCarthy C, Carey BC, Nowell-Bostic M, et al. Differentia diagnosis of pulmonary alveolar proteinosis: lung biopsy or blood test? Am J Respir Crit Care Med 2020; 201: A1093.

31. Carey B, Heald C, Chalk C, et al. Use of serum GM-CSF for diagnosis of patients with hereditary pulmonary alveolar proteinosis. Am J Respir Crit Care Med 2013; 187: A2850.

32. Kusakabe Y, Uchida K, Hiruma T, et al. A standardized blood test for the routine clinical diagnosis of impaired GM-CSF signaling using flow cytometry. J Immunol Methods 2014; 413: 1-11.
33. Hamvas A, Deterding RR, Wert SE, et al. Heterogeneous pulmonary phenotypes associated with mutations in the thyroid transcription factor gene NKX2-1. Chest 2013; 144 794-804

34. Gower WA, Wert SE, Ginsberg JS, et al. Fatal familial lung disease caused by $A B C A 3$ deficiency without identified $A B C A 3$ mutations. J Pediatr 2010; 157: 62-68.

35. Selman $M$, Lin HM, Montano $M$, et al. Surfactant protein $A$ and $B$ genetic variants predispose to idiopathic pulmonary fibrosis. Hum Genet 2003; 113: 542-550.

36. Agarwal PP, Seely JM, Perkins DG, et al. Pulmonary alveolar proteinosis and end-stage pulmonary fibrosis: a rare association. J Thorac Imaging 2005; 20: 242-244.

37. Campo I, Luisetti M, Griese M, et al. Whole lung lavage therapy for pulmonary alveolar proteinosis: a global survey of current practices and procedures. Orphanet J Rare Dis 2016; 11: 115.

38. Papiris SA, Tsirigotis $P$, Kolilekas L, et al. Long-term inhaled granulocyte macrophage-colony-stimulating factor in autoimmune pulmonary alveolar proteinosis: effectiveness, safety, and lowest effective dose. Clin Drug Investig 2014; 34 553-564.

39. Piloni D, Campo I. Current management strategies and the potential of inhaled GM-CSF for the treatment of autoimmune pulmonary alveolar proteinosis. Expert Opin Orphan Drugs 2019; 7: 117-123.

40. Sheng $G$, Chen $P$, Wei Y, et al. Better approach for autoimmune pulmonary alveolar proteinosis treatment: inhaled or subcutaneous granulocyte-macrophage colony-stimulating factor: a meta-analyses. Respir Res 2018; 19: 163.

41. Malur A, Kavuru MS, Marshall I, et al. Rituximab therapy in pulmonary alveolar proteinosis improves alveolar macrophage lipid homeostasis. Respir Res 2012; 13: 46.

42. Kavuru MS, Bonfield TL, Thomassen MJ. Plasmapheresis, GM-CSF, and alveolar proteinosis. Am J Respir Crit Care Med 2003; 167: 1036

43. Campo I. The influence of genetics on therapeutic developments in pulmonary alveolar proteinosis. Curr Opin Pulm Med 2019; 25: 294-299.

44. McCarthy C, Lee E, Bridges JP, et al. Statin as a nove pharmacotherapy of pulmonary alveolar proteinosis. Nat Commun 2018; 9: 3127. 